zu können, kam es zur Destabilisierung des gesamten Vergütungssystems. Zur Restabilisierung der Regelleistungsvolumen (RLV) wird die Honorarverteilung zum 1. Juli 2010 deshalb in einigen Punkten grundlegend verändert.

Künftig werden auch die aus der morbiditätsbedingten Gesamtvergütung (MGV) finanzierten „freien Leistungen“ (Vorwegleistungen, VWL) budgetiert. Diese Budgetierung erfolgt durch qualifikationsgebundene Zusatzvolumen (OZV) auf der Basis des Jahres 2008. Damit unterliegen $a b$ dem 1. Juli 2010 fast alle Leistungen der MGV einer Mengenbegrenzung. Davon nicht betroffen sind lediglich die bisher extrabudgetären Honorare für z.B. Präventionsleistungen und Impfungen, Disease-Management-Programme (DMP) oder Leistungen im Rahmen von ambulanten Operationen. Notfall- oder Laborleistungen und Kostenpauschalen werden weiterhin vorab vergütet, können aber auch budgetiert werden. Von der neuen Budgetierung nicht betroffen sind hingegen alle Leistungen, die bisher im Rahmen der Hausarztzentrierung nach $\S 73$ b SGB V abgeschlossen wurden.

\section{MMW Kommentar}

Da durch diese Maßnahmen zum 1.7.2010 kein zusätzliches Geld in das ambulante Versorgungssystem fließt, wird es zwangsläufig zu einer neuerlichen Umverteilung kommen, die sich allerdings in erster Linie innerhalb der einzelnen Fachgruppen abspielen wird. Ärzte, die bisher aus der Abrechnung "freier Leistungen" größere Umsätze erzielten, müssen mit Honorarverlusten rechnen.

Allerdings ist das keine Gesetzmäßigkeit, da die Änderung ein wichtiges Element enthält, die es fast jeder Praxis - zumindest im hausärztlichen Bereich - erlaubt, Verluste zu vermeiden.

Das budgetierte finanzielle Volumen, das der einzelnen Praxis ab Juli 2010 zur Verfügung steht, setzt sich nämlich aus dem bisher bekannten Regelleistungsvolumen (RLV) und dem neuen Qualitätszuschlags- volumen (QZV) zusammen. Beide Bereiche sind gegeneinander austauschbar, d.h. eine Unterdeckung im RLV kann durch Überschreitungen im QZV kompensiert werden und umgekehrt. Der Fallwert einer Praxis kann so gesehen erhalten bleiben. Gewinner oder Verlierer des neuen Systems generieren sich dann ausschlie $\beta$ lich in Abhängigkeit von der Fallzahlentwicklung. Verlieren können in erster Linie Praxen mit unterdurchschnittlicher Fallzahl, denen es nach dem neuen System nicht mehr möglich ist, über die "freien Leistungen "einen hohen Fallwert zu erzeugen, Gewinner werden Praxen mit höherer Fallzahl sein, die bisher wegen des niedrigen RLV-Fallwertes Verluste hinnehmen mussten. Der Umstand, dass sich im QZVBereich nunmehr aber auch Leistungen befinden, die bisher Bestandteil des RLV waren, eröffnet jedoch durchaus individuelle Perspektiven, die in den folgenden Ausgaben von $M M W$ einer näheren Betrachtung unterzogen werden.

\title{
Verstoßen Bonusregelungen bei der Arzneimittelverordnung gegen EU-Recht?
}

\begin{abstract}
— Zur Frage der Zulässigkeit von staatlich festgesetzten (finanziellen) Anreizmechanismen für Ärzte, bestimmte Arzneimittel zu verordnen, hat der EuGH-Generalanwalt seine Schlussanträge vorgelegt (C-62/o9). Der Verband der britischen Pharmaindustrie, ABPI, hatte Klage gegen eine Exekutivbehörde des britischen Gesundheitsministeriums (NHS) erhoben, die im Rahmen eines Vorabentscheidungsverfahrens durch den EuGH geprüft wird.

Zur Senkung der Arzneimittelkosten wurde ein Prämiensystem geschaffen, mit dem Ärzte dazu bewegt werden sollten, konkrete Arzneimittel zu verordnen. Dazu legt der NHS fest, welche Arzneimittel innerhalb derselben therapeutischen
\end{abstract}

Klasse, aber mit anderem Wirkstoff als gleichwertig anzusehen sind. Der ABPI hatte dagegen geklagt, da er der Auffassung ist, dass zur „Verkaufsförderung für Arzneimittel den zur Verschreibung oder Abgabe berechtigten Personen eine Prämie, finanzielle oder materielle Vorteile zu gewähren, anzubieten oder zu versprechen" verboten sei.

Der Generalanwalt ist der Ansicht, dass auch Behörden vom Geltungsbereich dieser Vorschrift erfasst werden und es einer öffentlichen Einrichtung somit nicht gestattet sei, zur Senkung ihrer Arzneimittelausgaben eine Regelung durchzuführen, in deren Rahmen Arztpraxen für die Verschreibung eines von der Anreizregelung erfassten Arzneimittels finanzielle
Anreize geboten bekommen. Die Schlussanträge des Generalanwalts sind für die EuGH-Richter nicht bindend.

\section{MMW Kommentar}

Abgesehen davon, dass es derartige Bonussysteme auch in Deutschland bereits gibt, sind die Pläne der Regierungskoalition zur Neuregulierung des Arzneimittelmarktes in diesem Zusammenhang beachtenswert.

Die Koalitionsfraktionen haben sich kürzlich über Eckpunkte zur Neustrukturierung des Arzneimittelmarktes abgestimmt und neben einer Verschlankung der Wirtschaftlichkeitsprüfungen auch den Einsatz von Bonus-Malus-Regelungen ins Auge gefasst. 